\title{
Current issues in gastric cancer management
}

\author{
Natalie G. Coburn
}

(C) The International Gastric Cancer Association and The Japanese Gastric Cancer Association 2012

This special issue on gastric cancer management is the first set of publications of a large body of work that involved a unique international collaboration. The primary interest of our group has been to examine processes of care for gastric cancer, to identify how patients are treated, and to determine the impact of variations in care on the outcome of surgery. This project began in 2008 with successful grant funding from the Canadian Cancer Society Research Institute (CCS-RI) to perform a RAND/UCLA Appropriateness Methodology (RAM) to examine complex issues in the care of gastric cancer patients. A major part of this study included the development of a multidisciplinary expert panel of 16 physicians from 6 countries representing 5 different sub-specialties, scoring over 2000 scenarios for appropriateness and necessity. In order to prepare this remarkable panel for the task at hand, a detailed review of the literature was undertaken. The goal was to synthesize the very latest evidence regarding processes of care for gastric cancer patients with a focus on peri-operative issues. This data could then be used by our expert panel as background for their scenario scoring.

The results of the wide-ranging literature reviews are presented in this special edition of Gastric Cancer. These systematic reviews and meta-analyses represent an overview of some of the most important issues in clinicaldecision making for surgeons and oncologists. What is striking, as one examines the vast literature on gastric cancer, is the paucity of randomized evidence, especially within the surgical setting. In some cases, the synthesis of all of the evidence still leaves us with more questions than answers. Indeed, encouraging more randomized controlled trials to improve our understanding of the disease and treatments was one of the issues that the panelists felt most strongly about during the consensus meeting in Toronto.

A project as large as this could not be completed without the help of numerous people and funding agencies. We are appreciative of the generous financial support obtained from the CCS-RI (operating grant \#019325), the Ministry of Health and Long Term Care of Ontario (scientific salary support), the Edmond Odette Cancer Centre, and the Sunnybrook Research Institute.

Our research team would like to extend special appreciation to Takeshi Sano, Yuko Kitagawa, and the individual reviewers for their encouragement and feedback. Additionally, we would like to express our gratitude to the thorough, tireless, and excellent staff at Gastric Cancer. It has been a privilege to lead this project, and I would like to offer heartfelt thanks to the members of the research team and our expert panelists. As well, I thank Taylor, Sophia, Nicholas, and Alexandra for their loving and constant support during this huge endeavor.

Finally, our team would like to dedicate this issue to Seana Rossi, whose strength, grace, and courage continue to be an inspiration to all of us.
N. G. Coburn ( $\square)$

Division of Surgical Oncology, Sunnybrook Health Sciences

Centre, Odette Cancer Centre, Suite T2-60, 2075 Bayview Ave,

Toronto, ON M4N 3M5, Canada

e-mail: natalie.coburn@sunnybrook.ca 